\title{
Cost-effectiveness analysis of universal noninvasive testing for post-treatment confirmation of Helicobacter pylori eradication and the impact of patient adherence
}

\author{
This article was published in the following Dove Press journal: \\ Patient Preference and Adherence \\ 8 June 2016 \\ Number of times this article has been viewed
}

\section{Susan H Boklage' \\ Allen W Mangel ${ }^{2}$ \\ Varun Ramamohan ${ }^{2}$ \\ Deirdre Mladsi \\ Tao Wang'}

'Otsuka America Pharmaceutical, Inc, Princeton, NJ, ${ }^{2}$ RTI Health Solutions, Research Triangle Park, NC, USA
Correspondence: Tao Wang

Department of Medical Affairs,

Otsuka America Pharmaceutical, Inc,

2440 Research Boulevard, Rockville,

MD 20850, USA

$\mathrm{Tel}+\mathrm{I} 2406833213$

Fax +13017217213

Email tao.wang@otsuka-us.com
Background: The treatment failure rate for Helicobacter pylori eradication therapy is $\sim 20 \%$ due to poor patient compliance and increased antibiotic resistance. This analysis assessed the cost-effectiveness of universal post-treatment testing to confirm eradication of $\mathrm{H}$. pylori infection in adults.

Methods: Decision-analytic models evaluated the cost-effectiveness of universal post-treatment testing (urea breath test [UBT] or monoclonal fecal antigen test [mFAT]) vs no testing (Model 1), and UBT vs mFAT after adjusting for patient adherence to testing (Model 2) in adults who previously received first-line antimicrobial therapy. Patients testing positive received secondline quadruple therapy; no further action was taken for those testing negative or with no testing (Model 1) or for those nonadherent to testing (Model 2). In addition to testing costs, excess lifetime costs and reduced quality-adjusted life-years (QALYs) due to continuing H. pylori infection were considered in the model.

Results: Expected total costs per patient were higher for post-treatment testing (UBT: US\$325.76; mFAT: US\$242.12) vs no testing (US\$182.41) in Model 1 and for UBT (US\$336.75) vs mFAT (US\$326.24) in Model 2. Expected QALYs gained per patient were 0.71 and 0.72 for UBT and mFAT, respectively, vs no testing (Model 1), and the same was 0.37 for UBT vs mFAT (Model 2). The estimated incremental costs per QALY gained for post-treatment testing vs no testing were US\$82.90-US\$202.45 and, after adjusting for adherence, US\$28.13 for UBT vs mFAT.

Conclusion: Universal post-treatment testing was found to be cost-effective for confirming eradication of $H$. pylori infection following first-line therapy. Better adherence to UBT relative to mFAT was the key to its cost-effectiveness.

Keywords: health-economic, decision-analytic modeling, infectious disease

\section{Introduction}

Helicobacter pylori infection is a common chronic infection that is associated with upper gastrointestinal diseases, including chronic gastritis, peptic ulcers, and gastric cancer. ${ }^{1}$ Individuals infected with $H$. pylori have a $15 \%$ lifetime risk of developing peptic ulcer disease and are ten times more likely to develop gastric adenocarcinoma than those who are not infected. ${ }^{2,3}$ The recommended first-line treatment for $H$. pylori infection according to the American College of Gastroenterology (ACG) practice guidelines is clarithromycin-based triple therapy, consisting of a proton pump inhibitor, clarithromycin, and either amoxicillin or metronidazole, or alternatively, bismuth quadruple therapy, consisting of bismuth, a proton pump inhibitor or histamine-2-receptor 
antagonist, metronidazole, and tetracycline. ${ }^{1}$ Approximately one in five cases of $H$. pylori infection is not eradicated by first-line therapy. ${ }^{4-6}$

Poor patient adherence and increased antibiotic resistance are the most important factors associated with failure of first-line therapy. ${ }^{1}$ In one US study of 125 patients with peptic ulcer disease or dyspepsia, $\sim 10 \%$ of patients failed to take $>60 \%$ of their $H$. pylori medications and $30 \%$ took $<90 \%$ of their medications. ${ }^{7}$ Patients who took $>60 \%$ of their prescribed medications had a $96 \%$ eradication rate compared with $69 \%$ for those who took $<60 \%{ }^{8}$ However, patient adherence to therapy is difficult to assess outside the clinical trial setting.

Studies in the US show significant antibiotic resistance among $H$. pylori isolates. For example, data obtained from 347 clinical isolates of H. pylori, collected from 1998 to 2002 from eleven hospital study sites across the US in the Helicobacter pylori Antimicrobial Resistance Monitoring Project, showed that $29 \%$ of isolates were resistant to one antimicrobial agent and $5 \%$ were resistant to $\geq 2$ agents. There was significant resistance to clarithromycin (13\%) and metronidazole $(25 \%)$, in addition to low resistance to amoxicillin (1\%). ${ }^{9}$ Eradication rates were significantly lower in patients with clarithromycin-resistant strains than in patients with clarithromycin-susceptible strains ( $29 \%$ vs 95\%) when triple therapy was used. ${ }^{10}$ Unfortunately, it is difficult to predict whether a patient would respond to the first-line therapy in clinical practice because H. pylori antibiotic sensitivity tests are not widely available in the US. ${ }^{1}$ Although dyspeptic symptoms tend to improve with first-line therapy, symptomatic relief is not a reliable predictor of eradication of $H$. pylori infection. ${ }^{11,12}$ Post-treatment testing is, therefore, essential to ensuring the successful eradication of $H$. pylori infection.

The ACG guidelines do not recommend universal testing following therapy to confirm eradication of $H$. pylori infection. ${ }^{1}$ These guidelines, however, mention specific situations where post-treatment testing of eradication of H. pylori infection is indicated, including in patients with $H$. pylori-associated ulcer or mucosa-associated lymphoid tissue lymphoma, persistent dyspeptic symptoms, and prior resection of early gastric cancer. These indications, except persistent dyspeptic symptoms, are associated with patients who have already developed $H$. pylori-related complications. The goal of this "test-and-treat" approach is to treat these patients earlier so that the risk of developing these complications can be reduced. Universal post-treatment testing to confirm eradication will further help to achieve this goal. When confirmation of eradication of $H$. pylori infection is deemed necessary, the guidelines designate the urea breath test (UBT) as the test of choice and the monoclonal fecal antigen test (mFAT) as an alternative. The polyclonal FAT does not have satisfactory sensitivity or positive predictive value in the post-treatment setting ${ }^{13}$ and, therefore, is not recommended for use in confirmation of eradication of H. pylori infection. ${ }^{1}$ Serologic antigen tests cannot distinguish between active H. pylori infection and previous exposure and, consequently, are not recommended for post-treatment testing.

The ACG guidelines recognize that "in an ideal world", all patients would undergo post-treatment testing to confirm eradication of $H$. pylori infection, but the guidelines also indicate that universal testing is neither practical nor cost-effective. ${ }^{1}$ No published economic analyses are cited, however, to support the assertion of a lack of cost-effectiveness. The widespread adoption of the UBT in clinical practice for confirming eradication of $H$. pylori infection is limited, in part, due to its higher cost relative to the mFAT. Thus, there is a need to assess the cost-effectiveness of universal testing of eradication of $H$. pylori infection following first-line therapy and to assess the cost-effectiveness of the UBT vs the mFAT.

Based on a targeted MEDLINE search using the keywords "H. pylori", "testing", and "cost-effectiveness", no primary clinical or modeling studies that assessed the economic value of tests used to confirm eradication of $H$. pylori infection after treatment were identified in the literature. One study showed a high incremental cost of the second UBT, with no incremental clinical benefit after the first post-treatment testing, but did not show the cost-effectiveness of post-treatment testing vs no testing. ${ }^{14}$ Of the previously published economic analyses of testing for $\mathrm{H}$. pylori infection, ${ }^{15-20}$ all but Davies et a ${ }^{16}$ considered only the costs of testing and treatment in their analyses and did not consider patient adherence to testing or the downstream costs of $H$. pylori-related diseases that a patient with continuing $H$. pylori infection is at an increased risk of developing (eg, the costs associated with managing gastric cancer, which a patient with continuing $H$. pylori infection is at an increased risk of developing $\sim 15$ years after diagnosis of infection). This underscores the need for a costeffectiveness analysis of post-treatment testing that incorporates patient adherence to testing, as well as assessment of long-term outcomes from continuing $H$. pylori infection.

The objectives of this study were as follows: 1) to compare the cost-effectiveness of universal post-treatment testing to confirm eradication of $H$. pylori infection following completion of first-line therapy vs no post-treatment testing (Model 1) and 2) to evaluate the cost-effectiveness of the 
UBT vs the mFAT, after adjusting for patient adherence to testing, for post-treatment testing to confirm eradication of H. pylori infection (Model 2).

\section{Methods}

Decision-analytic models were developed from a US thirdparty payer perspective to evaluate the cost-effectiveness of universal post-treatment testing vs no post-treatment testing to confirm eradication of $H$. pylori infection following completion of a first-line antimicrobial triple-therapy regimen (Figure 1), as well as to compare the cost-effectiveness of the UBT vs the mFAT in the post-treatment setting after adjusting for patient adherence to these tests (Figure 2). Only direct health care costs were considered in these models. Indirect costs, such as productivity lost due to treatment and other H. pylori-specific impairments, were not included. According to convention, patient copayments were not considered in the cost-effectiveness analyses.

The modeled study cohort comprised patients aged $\geq 18$ years (average age: 45 years) who had undergone one course of first-line therapy for eradication of $H$. pylori infection. In Model 1, patients were either tested (with the UBT or the mFAT) or not tested to confirm eradication of H. pylori infection after completing one course of antimicrobial therapy (Figure 1). In this model, patient adherence rates with the post-treatment tests were assumed to be $100 \%$.
Model 2 compared the UBT vs the mFAT in post-treatment testing following a course of first-line therapy, adjusting for adherence to the tests.

In Model 1, patients who tested positive - regardless of whether the result was a true or false positive - received second-line $H$. pylori eradication therapy. Patients who tested negative - regardless of whether the result was a false or true negative - and those who did not undergo posttreatment testing did not receive any additional eradication therapy. A similar approach was followed in Model 2. The UBT and the mFAT were compared as post-treatment tests to confirm eradication of $H$. pylori infection. Patients testing positive in these tests received second-line eradication therapy, whereas no further action was taken in the model for patients who were nonadherent to testing or for those who tested negative.

Excess lifetime costs (LTCs) and reduced quality-adjusted life-years (QALYs) - estimated with respect to patients without $H$. pylori infection - were applied to patients who continued to live with the infection. These patients included those with false-negative test results (Models 1 and 2), those who failed first-line therapy and opted not to undergo posttreatment testing (Model 1 only), those with true-positive test results who failed second-line therapy (Models 1 and 2), and those who were nonadherent to post-treatment testing (Model 2 only). Incremental cost-effectiveness

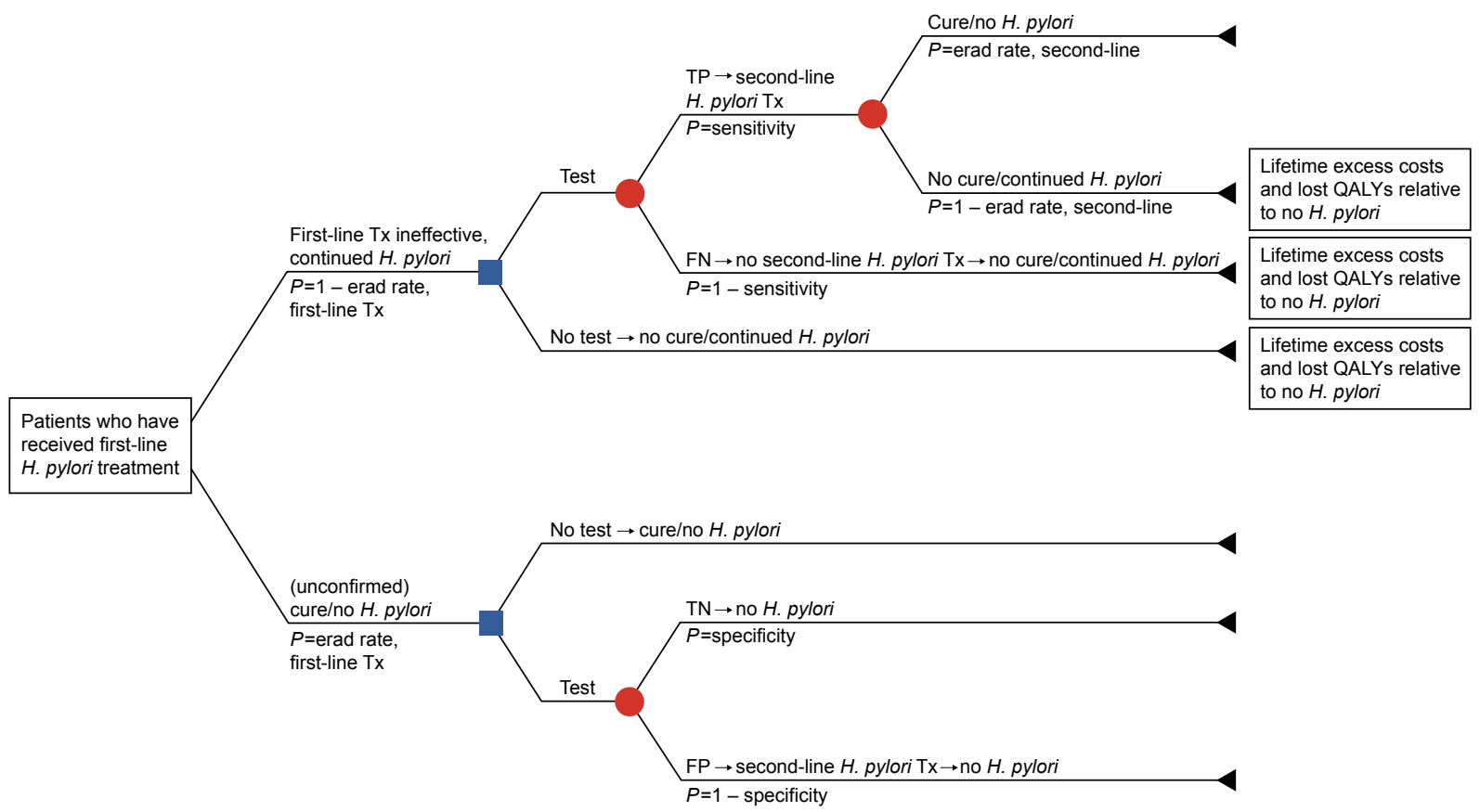

Figure I Decision-analytic model (Model I).

Abbreviations: erad, eradication; FN, false negative; FP, false positive; $H$. pylori, Helicobacter pylori; P, probability; QALYs, quality-adjusted life-years; TN, true negative; TP, true positive; Tx, treatment. 


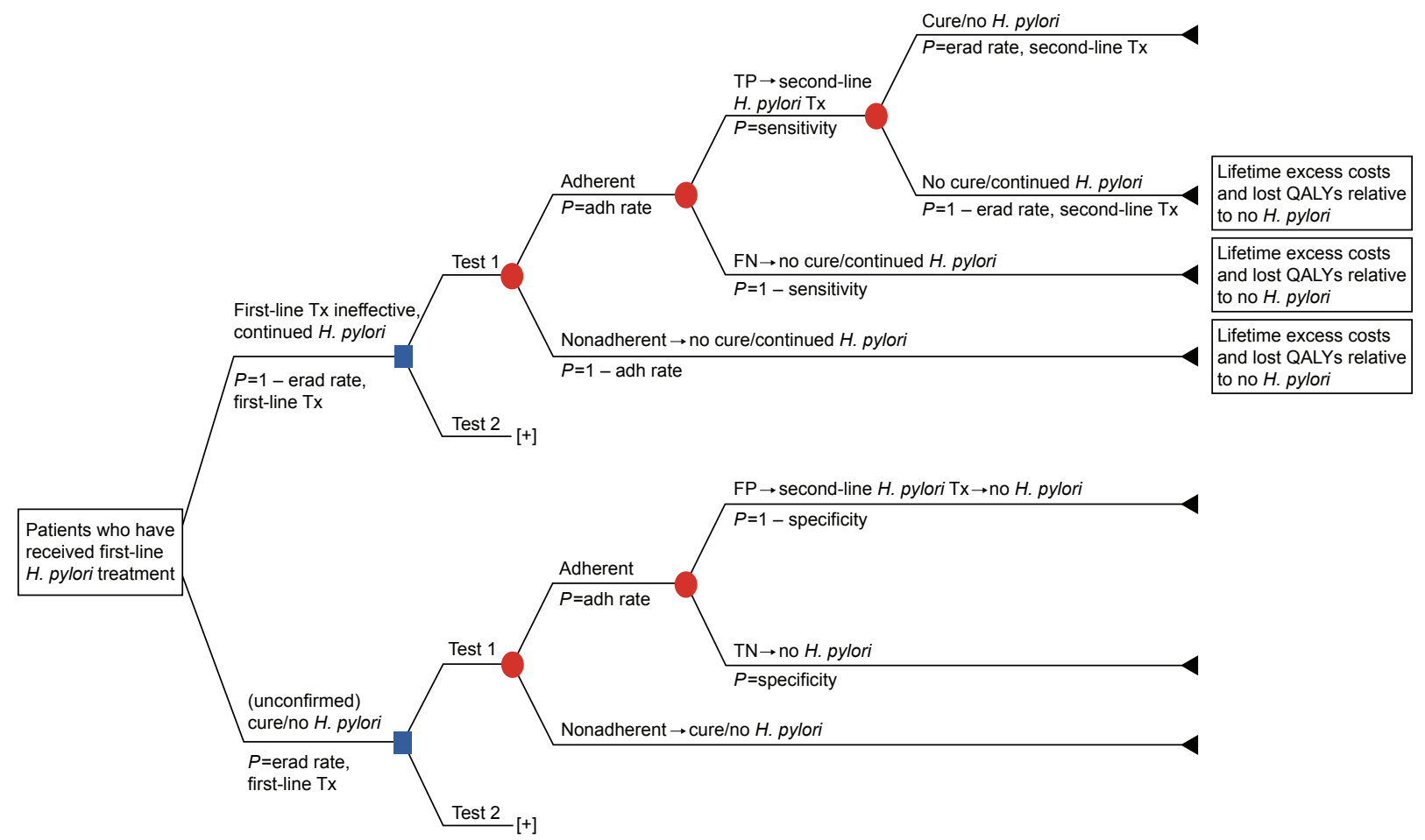

Figure 2 Decision-analytic model (Model 2).

Abbreviations: adh, adherence; erad, eradication; FN, false negative; FP, false positive; $H$. pylori, Helicobacter pylori; $P$, probability; QALYs, quality-adjusted life-years; TN, true negative; TP, true positive; Tx, treatment.

ratios (ICERs) were calculated from the incremental costs, and QALYs gained were estimated using the models.

Model input parameters for the UBT and the mFAT included their sensitivities, specificities, rates of test adherence, and costs (Table 1). ${ }^{13,21-23}$ The sensitivities and specificities of the UBT and the mFAT were estimated based on systematic reviews or meta-analyses evaluating these tests in post-treatment confirmation of eradication of $H$. pylori infection. ${ }^{13,22}$ The cost of each test was estimated based on the 2014 Resource-Based Relative Value Scale using the Current Procedural Terminology codes indicated in Table 1. Treatment parameter inputs to the model included costs and eradication rates of first-line triple therapy, as well as secondline quadruple therapy (Table 2). ${ }^{2-4,16,23-36}$ Patient adherence rates for the UBT and the mFAT ( $86 \%$ and $48 \%$, respectively) were obtained from the literature and were incorporated into

Table I Test parameters

\begin{tabular}{|c|c|c|c|}
\hline Parameter & Value & Variability, LB-UB & Source \\
\hline \multicolumn{4}{|l|}{ Test } \\
\hline \multicolumn{4}{|c|}{ UBT (CPT codes $83013+83014)$} \\
\hline Sensitivity, \% & 93.8 & $75.04-100.00$ & $\begin{array}{l}\text { Point estimate based on Gisbert and Pajares } 22 \text {; range for SA based on } \pm 20 \% \\
\text { (assumption; UB truncated at } 100 \% \text { ) }\end{array}$ \\
\hline Specificity, \% & 98.6 & $78.88-100.00$ & Same as UBT Sensitivity \\
\hline Adherence, \% ${ }^{\mathrm{a}}$ & 86.2 & $69.0-100.00$ & $\begin{array}{l}\text { Point estimate based on Cullen et } \mathrm{a}^{21} \text {; range for SA based on } \pm 20 \% \\
\text { (assumption; UB truncated at } 100 \% \text { ) }\end{array}$ \\
\hline Cost, US\$ & 102.81 & $82.25-123.37$ & Point estimate from RBRVS ${ }^{23}$; range for SA based on $\pm 20 \%$ (assumption) \\
\hline \multicolumn{4}{|c|}{ mFAT (CPT code 87338) } \\
\hline Sensitivity, \% & 96.0 & $76.00-100.00$ & $\begin{array}{l}\text { Point estimate from Gisbert and Pajares }{ }^{13} \text {; range for SA based on } \pm 20 \% \\
\text { (assumption; UB truncated at } 100 \% \text { ) }\end{array}$ \\
\hline Specificity, \% & 97.0 & $77.60-100.00$ & Same as mFAT Sensitivity \\
\hline Adherence, \% ${ }^{a}$ & 48.3 & $24 . I-72.4$ & Point estimate from Cullen et $\mathrm{a}^{21}$; range for SA based on $\sim \pm 50 \%$ (assumption) \\
\hline Cost, US\$ & 19.70 & I5.76-23.64 & Point estimate from RBRVS ${ }^{23}$; range for SA based on $\pm 20 \%$ (assumption) \\
\hline
\end{tabular}

Note: a Model 2 only.

Abbreviations: CPT, Current Procedure Terminology; LB, lower bound; mFAT, monoclonal fecal antigen test; RBRVS, Resource-Based Relative Value Scale; SA, sensitivity analysis; UB, upper bound; UBT, urea breath test. 
Table 2 Treatment, study cohort, and $H$. pylori disease-related parameters

\begin{tabular}{|c|c|c|c|}
\hline Parameter & Value & $\begin{array}{l}\text { Variability, } \\
\text { LB-UB }\end{array}$ & Source \\
\hline \multicolumn{4}{|l|}{ Triple therapy (first line) } \\
\hline Efficacy, \% & 81.0 & $77.00-85.00$ & $\begin{array}{l}\text { Point estimate based on Calvet et al }{ }^{4} \text {; range for SA based on } 95 \% \mathrm{Cl} \\
\text { reported in Calvet et al }{ }^{4}\end{array}$ \\
\hline Cost, US\$ & 205.85 & $164.68-247.02$ & $\begin{array}{l}\text { Point estimate based on Micromedex Red Book }{ }^{32} \text { for omeprazole, amoxicillin, } \\
\text { and clarithromycin for I } 4 \text { days; range for SA based on } \pm 20 \% \text { (assumption) }\end{array}$ \\
\hline \multicolumn{4}{|l|}{ Quadruple therapy (second line) } \\
\hline Efficacy, \% & 75.8 & $73.00-79.00$ & $\begin{array}{l}\text { Point estimate from Hojo et } \mathrm{a}^{28} \text {; range for } \mathrm{SA} \text { based on } 95 \% \mathrm{Cl} \text { reported in } \\
\text { Hojo et } \mathrm{a}^{28}\end{array}$ \\
\hline Cost, US\$ & 108.81 & $87.05-108.81$ & $\begin{array}{l}\text { Point estimate based on Micromedex Red Book }{ }^{32} \text { for omeprazole, } \\
\text { metronidazole, tetracycline, and bismuth for } 14 \text { days; range for SA based on } \\
\pm 20 \% \text { (assumption) }\end{array}$ \\
\hline \multicolumn{4}{|c|}{ Physician visit cost (CPT codes $99214+99244)$, US\$ } \\
\hline PCP visit & 107.83 & $86.26-129.40$ & Point estimate from RBRVS ${ }^{23}$; range for SA based on $\pm 20 \%$ (assumption) \\
\hline GI doctor visit & 185.92 & $148.74-223.10$ & Same as PCP visit \\
\hline \multicolumn{4}{|c|}{ H. pylori-related disease parameters } \\
\hline \multicolumn{4}{|c|}{ Lifetime $H$. pylori-positive outcome probability, \% } \\
\hline Gastric cancer & 1.0 & $0.50-1.50$ & Point estimate from Kuipers ${ }^{30}$; range for SA based on $\pm 50 \%$ (assumption) \\
\hline PUD & 15.0 & $10.00-20.00$ & $\begin{array}{l}\text { Point estimate from Peterson et al }{ }^{3} \text {; range for SA based on } \pm 33.33 \% \\
\text { (assumption) }\end{array}$ \\
\hline Dyspepsia & 100.0 & $80.00-100.00$ & Assumed \\
\hline \multicolumn{4}{|c|}{ Lifetime $H$. pylori-negative outcome probability, \% } \\
\hline Gastric cancer & 0.1 & $0.08-0.12$ & $\begin{array}{l}\text { Point estimate based on Kuipers }{ }^{30} \text { and Kusters et } a^{2} \text {; range for SA based on } \\
\pm 20 \% \text { (assumption) }\end{array}$ \\
\hline PUD & 3.8 & $3.00-4.50$ & $\begin{array}{l}\text { Point estimate based on Laine et } \mathrm{a}^{31} \text { and Peterson et } \mathrm{al}^{3} \text {; range for SA based } \\
\text { on } \pm 20 \% \text { (assumption) }\end{array}$ \\
\hline Dyspepsia & 16.5 & $6.00-24.00$ & $\begin{array}{l}\text { Point estimate based on Kusters et } \mathrm{al}^{2} \text {; range for SA based on range reported } \\
\text { in Kusters et } \mathrm{al}^{2}\end{array}$ \\
\hline \multicolumn{4}{|l|}{ Median survival, years } \\
\hline Gastric cancer & 1.71 & $1.37-2.05$ & $\begin{array}{l}\text { Point estimate based on Davies et al }{ }^{16} \text {; range for SA based on } \pm 20 \% \\
\text { (assumption) }\end{array}$ \\
\hline \multicolumn{4}{|l|}{ Disease duration } \\
\hline \multicolumn{4}{|c|}{ Average time elapsed between diagnosis of $H$. pylori infection and diagnosis of: } \\
\hline Gastric cancer, years & 15 & $12-18$ & $\begin{array}{l}\text { Point estimate based on Asaka et } \mathrm{a}^{25} \text { and Davies et } \mathrm{al}^{16} \text {; range for SA based } \\
\text { on } \pm 20 \% \text { (assumption) }\end{array}$ \\
\hline Dyspepsia, years & 1.55 & $1.24-1.86$ & $\begin{array}{l}\text { Point estimate based on Chiba et } \mathrm{al}^{26} ; \text { range for SA based on } \pm 20 \% \\
\text { (assumption) }\end{array}$ \\
\hline PUD, years & 0.5 & $0.25-0.75$ & Assumption based on discussion with clinical consultant \\
\hline Hospitalization due to PUD, days & 4.3 & $3.44-5.16$ & $\begin{array}{l}\text { HCUP with ICD-9 code of } 578.9 \text { (hemorrhage of GI tract); range for SA } \\
\text { based on } \pm 20 \% \text { (assumption) }\end{array}$ \\
\hline \multicolumn{4}{|l|}{ Utility values } \\
\hline Gastric cancer & 0.49 & $0.17-0.79$ & Yeh et $\mathrm{al}^{35} ;$ range for SA reported in Yeh et a ${ }^{35}$ \\
\hline PUD & 0.92 & $0.8 I-0.96$ & Howard et $\mathrm{a}^{29}$; range for SA reported in Howard et a ${ }^{29}$ \\
\hline GI hospitalization due to PUD & 0.50 & $0.40-0.60$ & Point estimate from Erstad ${ }^{27}$; range for SA based on $\pm 20 \%$ (assumption) \\
\hline Chronic dyspepsia & 0.97 & $0.74-0.98$ & Point estimate from You et $\mathrm{al}^{36}$; range for SA reported in You et al ${ }^{36}$ \\
\hline \multicolumn{4}{|l|}{ Associated costs, US\$ } \\
\hline Gastric cancer & 47,578 & $43,723-5 I, 342$ & Yabroff et $\mathrm{a}^{34} ;$ range based on $95 \% \mathrm{Cl}$ reported in Yabroff et $\mathrm{al}^{34}$ \\
\hline PUD managed with medication (/year) & 481 & $385-578$ & Estimated based on Slawsky et al ${ }^{33}$; range for SA based on $\pm 20 \%$ (assumption) \\
\hline Hospitalization due to PUD & 8,896 & $7,117-10,675$ & HCUP with ICD-9 code of 578.9 ; range for SA based on $\pm 20 \%$ (assumption) \\
\hline Dyspepsia (/year) & 481 & $385-578$ & Estimated based on Slawsky et $\mathrm{al}^{33}$; range for SA based on $\pm 20 \%$ (assumption) \\
\hline \multicolumn{4}{|l|}{ Reduced QALYs and excess costs } \\
\hline Expected life-years at age 45 years & 34.34 & $27.47-4 \mid .21$ & Point estimate from Arias et $\mathrm{a}^{24}$; range for $\mathrm{SA}$ based on $\pm 20 \%$ (assumption) \\
\hline Estimated reduced QALYs & 5.35 & $3.4 \mathrm{I}-7.98$ & $\begin{array}{l}\text { Point estimate derived using } H \text {. pylori-related disease parameters listed below; } \\
\text { range for SA estimated from Monte Carlo simulations (generated by sampling } \\
\text { from intervals of uncertainty around } H \text {. pylori-related disease parameters) }\end{array}$ \\
\hline Estimated excess LTCs, US\$ & 960 & $679.27-1,125.89$ & Same as Estimated reduced QALYs \\
\hline
\end{tabular}

Abbreviations: $\mathrm{Cl}$, confidence interval; CPT, Current Procedure Terminology; Gl, gastrointestinal; HCUP, Healthcare Cost and Utilization Project; ICD, International Classification of Diseases; LB, lower bound; LTC, lifetime costs; PCP, primary care physician; PUD, peptic ulcer disease; QALYs, quality-adjusted life-years; RBRVS, ResourceBased Relative Value Scale; SA, sensitivity analysis; SE, standard error; UB, upper bound; H. pylori, Helicobacter pylori. 
this model. ${ }^{21}$ Utility estimates that reflect the health-related quality of life associated with developing $H$. pylori-related diseases were obtained from the literature and were combined with estimates of disease duration and survival obtained from the literature to generate QALYs. ${ }^{27,29,35,36}$ (Formally, utility estimates are preference weights, measured on a scale of $0-1$, where zero indicates death and one indicates full health, which reflect an individual's preferences for different health outcomes.) $)^{37}$

Excess LTCs and reduced QALYs due to continuing $H$. pylori infection were estimated for the following $H$. pylorirelated diseases: gastric cancer, peptic ulcer disease, and continuing dyspepsia. Excess LTCs for each disease were estimated separately and then summed to obtain the total expected per-patient excess LTCs due to continuing $H$. pylori infection. The estimation of excess LTCs due to continuing $H$. pylori infection is shown in the following equations:

$$
\mathrm{LTC}_{H P}=\sum_{i \in\{\mathrm{GC}, \text { PUD, Dysp }\}} \mathrm{LTC}_{i}
$$

where $\mathrm{LTC}_{H P}$ stands for excess LTCs due to continuing H. pylori infection; GC is gastric cancer; PUD indicates peptic ulcer disease; Dysp indicates dyspepsia; and $\mathrm{LTC}_{i}$ stands for excess LTCs for each $H$. pylori-related disease.

Excess LTCs for each of the three H. pylori-related diseases were in turn estimated as the per-patient cost of managing each disease multiplied by the excess risk associated with developing each disease due to continuing $H$. pylori infection:

$$
\mathrm{LTC}_{i}=\left(P_{i[w]}-P_{i[w o]}\right) \cdot C_{i}
$$

where $P_{i}$ is the risk associated with developing each disease; $[w]$ is the condition with $H$. pylori infection; [wo] indicates condition without $H$. pylori infection; and $C_{i}$ is the cost for treating each disease.

The per-patient cost of managing peptic ulcer disease was estimated as the sum of the cost of peptic ulcer disease managed by medication (regardless of hospitalization) and the cost of a related hospitalization multiplied by its probability of occurrence:

$$
C_{P U D}=\left(t_{P U D} \cdot C_{P U D[m e d]}+P_{P U D[h o s p]} \cdot C_{P U D[h o s p]}\right)
$$

where $C_{P U D}$ is the cost per patient of managing peptic ulcer disease; $t_{P U D}$ is the duration of peptic ulcer disease (in years);
$C_{P U D[\mathrm{med}]}$ is the cost per patient per year of managing peptic ulcer disease by medication (regardless of hospitalization); $C_{\text {PUD hosp }]}$ represents the cost of related hospitalization; and $P_{P U D[h o s p]}$ is its probability of occurrence.

The per-patient cost of managing dyspepsia was estimated as the duration of dyspepsia multiplied by the annual per-patient cost of managing dyspepsia. It was assumed that all patients with continuing $H$. pylori infection who did not develop peptic ulcer disease or gastric cancer developed dyspepsia:

$$
\begin{gathered}
C_{D y s p}=t_{D y s p} \cdot C_{D y s p[a n n]} \\
P_{\text {Dysp[w] }}=1-P_{\mathrm{GC}[\mathrm{w}]}-P_{\mathrm{PUD}[\mathrm{w}]}
\end{gathered}
$$

where $C_{D y s p}$ is the cost per patient of managing dyspepsia; $t_{D y s p}$ represents the duration of dyspepsia (in years); $C_{D y s p[a n n]}$ is the annual cost per patient of managing dyspepsia; and $P$ is the probability of occurrence.

Estimates of the reduced QALYs associated with continuing $H$. pylori infection were derived in a similar manner. As an illustration of the application of the aforementioned equations, the estimation of excess LTCs associated with peptic ulcer disease is described in a step-by-step manner in Table S1.

Estimates of uncertainty for the input parameters (eg, standard errors and ranges) were obtained from the source publications where available; if not, assumptions were made (Tables 1 and 2). For the probabilistic sensitivity analyses, the $\beta$-distribution was used for the sensitivities, specificities, and adherence rates of the tests, as well as all other probabilities used in the model. The Gaussian distribution is used for all costs. To characterize the uncertainty associated with the estimated excess LTCs and reduced QALYs, a Monte Carlo simulation addressing first- and second-order uncertainties was performed. The mean and standard deviations of these parameters were estimated from a sufficiently large number of values of the excess LTCs and reduced QALYs generated by the Monte Carlo simulation. These descriptive statistics then were used to generate lower and upper bounds for the one-way sensitivity analyses. For the probabilistic sensitivity analyses, appropriate distributions were selected based on the resulting simulated data sets. Excess LTCs and reduced QALYs were estimated for patients aged 45 years, which was representative of the study cohort in this modeling exercise and is near the midpoint of the age range of an adult population, with 77 years being the average US life expectancy. ${ }^{24}$ The estimate of expected life-years remaining was allowed to vary in the Monte Carlo simulations. 


\section{Results}

In the base-case scenario for Model 1, in which patient adherence to post-treatment testing was assumed to be $100 \%$ for both the UBT and the mFAT, the total costs for each treated patient were US\$325.76 for the UBT, US\$242.12 for the mFAT, and US\$182.41 for no post-treatment testing (Table 3). The higher total costs associated with the UBT vs the mFAT reflected higher test costs. It is important to recognize, however, that adherence rates with these respective noninvasive tests were not incorporated into Model 1.

The QALYs gained per patient with the UBT and the mFAT compared with no post-treatment testing were 0.71 and 0.72 , respectively. The ICERs were US\$202.45 with the UBT and US $\$ 82.90$ with the mFAT compared with no posttreatment testing (Table 3; Figure 3). Thus, providing a noninvasive post-treatment test was a cost-effective alternative to not testing for eradication of $H$. pylori infection following completion of first-line therapy. The higher costs associated with testing (ie, costs of tests, physician visits, and secondline eradication therapy: US\$270.32 for UBT and US\$188.86 for mFAT) were partially offset by decreases in the LTCs incurred from continuing $H$. pylori infection compared with no post-treatment testing (US\$55.45 for UBT and US\$53.26 for mFAT vs US\$182.41 for no testing; Table 3).

In the base-case scenario for Model 2, in which the estimated adherence rates were $86 \%$ for the UBT and $48 \%$ for the mFAT, the corresponding calculated total costs per patient were US $\$ 336.75$ and US\$326.24 (Table 4). In this model, the higher test costs associated with the UBT were

Table 3 Base-case outcomes for Model I

\begin{tabular}{|c|c|c|c|}
\hline Outcome & UBT & mFAT & $\begin{array}{l}\text { No } \\
\text { retest }\end{array}$ \\
\hline \multicolumn{4}{|l|}{ Economic outcomes per patient, US\$ } \\
\hline Total costs & 325.76 & 242.12 & $|82.4|$ \\
\hline Tests & 102.81 & 19.70 & 0.00 \\
\hline Physician visits & 146.88 & 146.88 & 0.00 \\
\hline Second-line eradication & 20.63 & 22.28 & 0.00 \\
\hline Excess lifetime costs & 55.45 & 53.26 & $|82.4|$ \\
\hline \multicolumn{4}{|l|}{ Incremental costs } \\
\hline Costs per accurately diagnosed case, US\$ & 333.47 & 250.59 & 0.00 \\
\hline ICER, US\$/QALY gained vs no retest & 202.45 & 82.90 & - \\
\hline Cost-effective vs no retest? & Y & Y & - \\
\hline \multicolumn{4}{|l|}{ Health outcomes per patient } \\
\hline Cases accurately diagnosed as positive (n) & 0.18 & 0.18 & 0.00 \\
\hline Cases accurately diagnosed as negative (n) & 0.80 & 0.79 & 0.00 \\
\hline Reduced QALYs due to continuing & 0.31 & 0.30 & 1.02 \\
\hline H. pylori infection & & & \\
\hline
\end{tabular}

Abbreviations: ICER, incremental cost-effectiveness ratio; mFAT, monoclona fecal antigen test; QALYs, quality-adjusted life-years; UBT, urea breath test; Y, yes: H. pylori, Helicobacter pylori. nearly fully offset by the higher LTC associated with the mFAT, reflecting the significant difference in adherence rates between the two tests. Notably, the cost per accurately diagnosed case following first-line triple therapy was $\sim 75 \%$ higher with the mFAT vs the UBT. Expected QALY gained per patient for the UBT vs the mFAT was 0.37 . The UBT was cost-effective as a post-treatment test compared with the mFAT. After adjusting for patient adherence, the ICER of the UBT was US\$28.13/QALY gained compared with the mFAT for testing to confirm eradication of $H$. pylori infection (Figure 3).

One-way sensitivity analyses indicated that none of the model parameters were influential in terms of changing the cost-effectiveness conclusions in either Model 1 or Model 2. In the case of Model 2, when the UBT was compared with the mFAT, the results of the one-way sensitivity analysis indicated that when the cost of the UBT was set to its lower bound or the adherence rate with the UBT was set to its upper bound, the UBT was cost saving. Similarly, when the H. pylori infection eradication rate of first-line treatment was set to its lower bound, the UBT was cost saving. Probabilistic sensitivity analyses indicated that the cost-effectiveness of both the UBT and the mFAT was highly robust to uncertainty in the model parameters when used in the post-treatment testing, with the probabilities of cost-effectiveness being 1.00 for both tests compared with no post-treatment testing at a base-case willingness-to-pay threshold of US\$50,000/ QALY gained for the ICER. The results of the probabilistic sensitivity analysis for the comparison between the UBT and the mFAT (after adjusting for adherence) are illustrated as a scatterplot in Figure 4. The UBT was cost-effective or cost saving in all scenarios evaluated in the probabilistic sensitivity analysis, as seen from the presence of all points in the scatterplot being in the first quadrant below the threshold (not shown due to limitations in figure size) or in the fourth quadrant.

\section{Discussion}

The current ACG practice guidelines assert that universal post-treatment testing for confirming eradication of $H$. pylori infection following first-line triple therapy is not cost-effective, but they do not provide any economic data to support this conclusion. ${ }^{1}$ This study evaluated the costeffectiveness of universal post-treatment testing using two noninvasive tests (the UBT and the mFAT). Both noninvasive tests are recommended in the AGA guidelines for posttreatment confirmation of eradication of $H$. pylori infection in specific types of patients. 


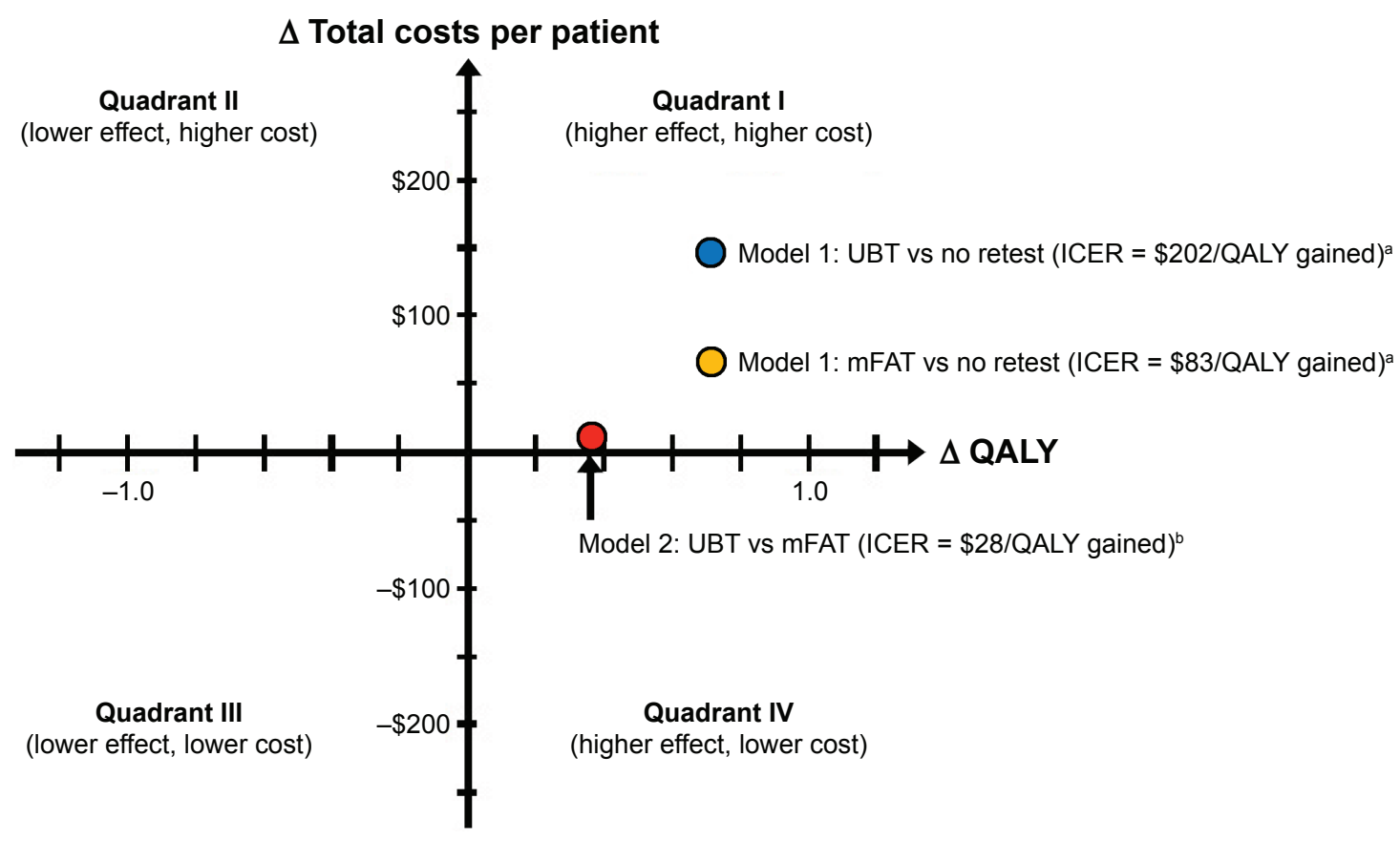

Figure 3 Cost-effectiveness of universal post-treatment testing.

Notes: a $100 \%$ patient adherence to testing. ${ }^{\mathrm{b}} 86 \%$ adherence to UBT and $48 \%$ adherence to mFAT.

Abbreviations: ICER, incremental cost-effectiveness ratio; QALY, quality-adjusted life-year; UBT, urea breath test; mFAT, monoclonal fecal antigen test.

H. pylori infection is a common chronic infection with a seroprevalence of $27 \%$ in the US. ${ }^{38}$ This disease can lead to more severe long- and short-term consequences (eg, gastric cancer and peptic ulcer disease) if it is not eradicated. Testing to confirm eradication after a course of antimicrobial treatment is critical for the following reasons: 1) the recurrence rate of $H$. pylori infection after successful eradication is a low $2.5 \% /$ year in the US; $; 9$ and 2) treatment failure rates have

Table 4 Base-case outcomes for Model 2

\begin{tabular}{lll}
\hline Outcome & UBT & mFAT \\
\hline Economic outcomes per patient, US\$ & & \\
Total costs & 336.75 & 326.24 \\
Tests & 88.62 & 9.52 \\
Physician visits & 146.88 & 146.88 \\
Second-line eradication & 20.63 & 22.28 \\
Excess lifetime costs & 80.63 & 147.57 \\
Incremental costs & & \\
Costs per accurately diagnosed case, US\$ & 399.91 & 699.08 \\
ICER, US\$/QALY gained (UBT vs mFAT) & - & 28.13 \\
$\quad$ UBT cost-effective vs mFAT? & - & Y \\
Health outcomes per patient & & \\
Cases accurately diagnosed as positive (n) & 0.15 & 0.09 \\
Cases accurately diagnosed as negative (n) & 0.69 & 0.38 \\
Reduced QALYs due to continuing & 0.45 & 0.82 \\
H. pylori infection & & \\
\hline Abbreviations: ICER, ing & & \\
\hline
\end{tabular}

Abbreviations: ICER, incremental cost-effectiveness ratio; mFAT, monoclonal fecal antigen test; QALYs, quality-adjusted life-years; UBT, urea breath test; Y, yes; H. pylori, Helicobacter pylori. been found to be high due to increasing antibiotic resistance. ${ }^{40}$ Specifically, a global review of clinical trial data showed that only $18 \%$ of trials demonstrated eradication rates $>85 \%$ for first-line triple therapy, and $\sim 60 \%$ of trials had eradication rates $<80 \%$ by intent-to-treat analyses. ${ }^{40}$ Failure rates are likely to be even higher outside the clinical trial setting due to poor patient adherence to the treatment regimen.

This cost-effectiveness analysis incorporated the downstream costs of continuing H. pylori infection (both Models 1 and 2), as well as patient adherence to the two noninvasive tests (Model 2 only). Using LTCs that consider the increased

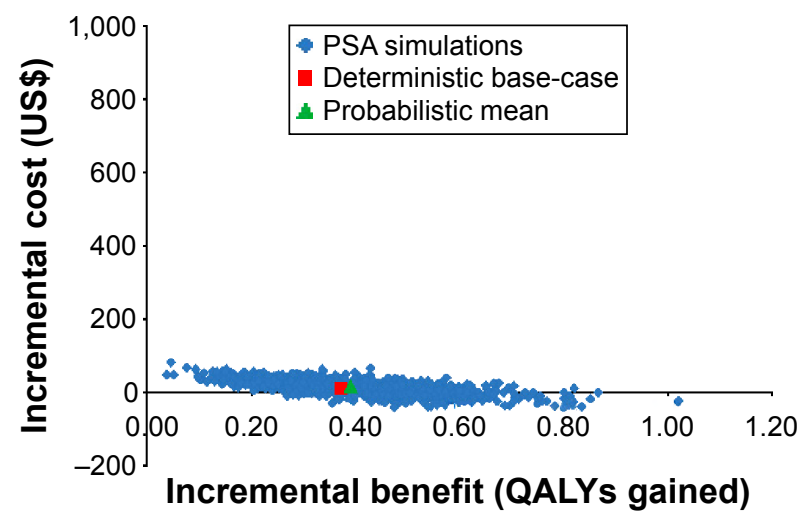

Figure 4 Cost-effectiveness scatterplot for comparison of urea breath test with the monoclonal fecal antigen test after adjusting for adherence.

Abbreviations: PSA, probabilistic sensitivity analysis; QALYs, quality-adjusted life-years. 
expected downstream costs (resulting from the increased risks of developing gastric cancer, peptic ulcer disease, and continuing dyspepsia) due to continuing $H$. pylori infection is a more comprehensive approach than only considering short-term costs (eg, physician visits, testing, and treatment costs) used in other cost-effectiveness reports in the context of eradication of $H$. pylori infection. Furthermore, the cost of confirming eradication is unlikely to be a recurring expense due to the low risk of recurrence of $H$. pylori infection after a successful eradication confirmed by the UBT. ${ }^{39}$

In this study, the results from Model 1 demonstrated that universal post-treatment testing of eradication of $H$. pylori infection following first-line therapy was cost-effective compared with no post-treatment testing, whereas the results from Model 2 showed that post-treatment testing with the UBT following first-line therapy was cost-effective compared with the mFAT after adjusting for patient adherence to testing. Universal post-treatment testing was found to be costeffective, at a modest increase in costs compared with the ICER threshold. Furthermore, the UBT yielded substantially better outcomes at a modest increase in costs compared with the mFAT, after adjusting for patient adherence to testing.

The results of this cost-effectiveness analysis need to be interpreted cautiously due to the assumptions underlying the model structure, as well as the input parameters to the models. The primary limitation originates from the assumptions made for parameters for which data were not available in the literature or for which only limited data were available. Although these assumptions were confirmed as being realistic by a clinical consultant, as well as being tested and found to be robust in sensitivity analyses, it would be prudent to update the parameter estimates as additional information becomes available in the literature. Specifically, although patient adherence to testing was the key driver of the costeffectiveness of the UBT, compared with the mFAT, limited adherence data are available in the literature. This was not, however, an issue in the evaluation of the cost-effectiveness of universal post-treatment testing vs no testing because adherence data were not included in Model 1.

\section{Conclusion}

Universal post-treatment testing using the UBT and the mFAT following first-line therapy was found to be costeffective in confirming eradication of $H$. pylori infection compared with no post-treatment testing. Moreover, the UBT was a cost-effective option in this setting compared with the mFAT after adjusting for patient adherence to testing. When both models were considered together, the UBT was shown to improve health outcomes at a modest increase in total costs, compared with no testing or testing with the mFAT. Notably, the higher test cost of the UBT within the range of current reimbursement schemes was not a factor that affected the outcome of this cost-effectiveness analysis.

\section{Acknowledgments}

This research was fully funded by Otsuka America Pharmaceutical, Inc. Editorial assistance was provided by Catherine Fontana, Geoff Marx, and Barry M Weichman, PhD, of BioScience Communications, New York, NY, which was also funded by Otsuka America Pharmaceutical, Inc.

The abstract of this manuscript was presented as a poster presentation at the 79th Annual Scientific Meeting of the American College of Gastroenterology, held in October 2014 at Philadelphia. The abstract was published in The American Journal of Gastroenterology, Vol 109, Supplement 2, Page s662, October 2014 (http://www.nature.com/ajg/journal/ v109/n2s/pdf/ajg2014280a.pdf).

\section{Disclosure}

SHB is a former employee of Otsuka. AWM, VR, and DM are employees of RTI, which was contracted by Otsuka to conduct this study. TW is an employee of Otsuka. The authors report no other conflicts of interest in this work.

\section{References}

1. Chey WD, Wong BC; Practice Parameters Committee of the American College of Gastroenterology. American College of Gastroenterology guideline on the management of Helicobacter pylori infection. Am J Gastroenterol. 2007;102:1808-1825.

2. Kusters JG, van Vliet AHM, Kuipers EJ. Pathogenesis of Helicobacter pylori infection. Clin Microbiol Rev. 2006;19:449-490.

3. Peterson WL, Fendrick AM, Cave DR, Peura DA, Garabedian-Ruffalo SM, Laine L. Helicobacter pylori-related disease. Guidelines for testing and treatment. Arch Intern Med. 2000;160:1285-1291.

4. Calvet X, García N, López T, Gisbert JP, Gené E, Roque M. A metaanalysis of short versus long therapy with a proton pump inhibitor, clarithromycin and either metronidazole or amoxycillin for treating Helicobacter pylori infection. Aliment Pharmacol Ther. 2000;14: 603-609.

5. Katelaris PH, Forbes GM, Talley NJ, Crotty B. A randomized comparison of quadruple and triple therapies for Helicobacter pylori eradication: the QUADRATE study. Gastroenterology. 2002;123:1763-1769.

6. Vakil N, Lanza F, Schwartz H, Barth J. Seven-day therapy for Helicobacter pylori in the United States. Aliment Pharmacol Ther. 2004;20: 99-107.

7. Lee M, Kemp JA, Canning A, Egan C, Tataronis G, Farraye FA. A randomized controlled trial of an enhanced patient compliance program for Helicobacter pylori therapy. Arch Intern Med. 1999;159:2312-2316.

8. Graham DY, Lew GM, Malaty HM, et al. Factors influencing the eradication of Helicobacter pylori with triple therapy. Gastroenterology. 1992; 102:493-496.

9. Duck WM, Sobel J, Pruckler JM, et al. Antimicrobial resistance incidence and risk factors among Helicobacter pylori-infected persons, United States. Emerg Infect Dis. 2004;10:1088-1094. 
10. Vaira D, Zullo A, Vakil N, et al. Sequential therapy versus standard triple-drug therapy for Helicobacter pylori eradication. Ann Intern Med. 2007;146:556-563.

11. Gisbert JP, Calvet X. Helicobacter pylori "test-and-treat" strategy for management of dyspepsia: a comprehensive review. Clin Transl Gastroenterol. 2013;4:e32.

12. Jaakkimainen RL, Boyle E, Tudiver F. Is Helicobacter pylori associated with non-ulcer dyspepsia and will eradication improve symptoms? A meta-analysis. BMJ. 1999;319:1040-1044.

13. Gisbert JP, Pajares JM. Stool antigen test for the diagnosis of Helicobacter pylori infection: a systematic review. Helicobacter. 2004;9: 347-368.

14. Vakil N, Zullo A, Ricci C, Hassan C, Vaira D. Duplicate breath testing to confirm eradication of Helicobacter pylori: incremental benefit and cost in 419 patients. Aliment Pharmacol Ther. 2008;28:1304-1308.

15. Chey WD, Fendrick AM. Noninvasive Helicobacter pylori testing for the test-and-treat strategy: a decision analysis to assess the effect of past infection on test choice. Arch Intern Med. 2001;161:2129-2132.

16. Davies R, Crabbe D, Roderick P, Goddard JR, Raftery J, Patel P. A simulation to evaluate screening for Helicobacter pylori infection in the prevention of peptic ulcers and gastric cancers. Health Care Manag Sci. 2002;5:249-258.

17. Elwyn G, Taubert M, Davies S, Brown G, Allison M, Phillips C. Which test is best for Helicobacter pylori? A cost-effectiveness model using decision analysis. Br J Gen Pract. 2007;57:401-403.

18. Holmes KP, Fang JC, Jackson BR. Cost-effectiveness of six strategies for Helicobacter pylori diagnosis and management in uninvestigated dyspepsia assuming a high resource intensity practice pattern. $B M C$ Health Serv Res. 2010;10:344.

19. Masucci L, Blackhouse G, Goeree R. Cost-effectiveness of the carbon-13 urea breath test for the detection of Helicobacter pylori: an economic analysis. Ont Health Technol Assess Ser. 2013;13:1-28.

20. Vakil N, Rhew D, Soll A, Ofman JJ. The cost-effectiveness of diagnostic testing strategies for Helicobacter pylori. Am J Gastroenterol. 2000; 95:1691-1698.

21. Cullen KP, Broderick BM, Jayaram J, Flynn B, O’Connor HJ. Evaluation of the Helicobacter pylori stool antigen ( $\mathrm{HpSA}$ ) test in routine clinical practice - is it patient-friendly? Ir Med J. 2002;95:305-306.

22. Gisbert JP, Pajares JM. ${ }^{13} \mathrm{C}$-urea breath test in the diagnosis of Helicobacter pylori infection - a critical review. Aliment Pharmacol Ther. 2004;20:1001-1017.

23. Resource-Based Relative Value Scale (RBRVS). The Essential RBRVS 2014: A Comprehensive Listing of RBRVS Values for CPT and HCPCS Codes. Eden Prairie, MN: OptumInsight, Inc; 2014

24. Arias E, Curtin LR, Wei R, Anderson RN. U.S. decennial life tables for 1999-2001, United States life tables. Natl Vital Stat Rep. 2008;57: $1-36$.

25. Asaka M, Sepulveda AR, Sugiyama T, Graham DY. Gastric cancer. In: Mobley HLT, Mendz GL, Hazell SL, editors. Helicobacter pylori: Physiology and Genetics. Washington, DC: American Society for Microbiology (ASM) Press; 2001. Available from: http://www.ncbi. nlm.nih.gov/books/NBK2445/. Accessed March 6, 2014.
26. Chiba N, Van Zanten SJ, Sinclair P, Ferguson RA, Escobedo S, Grace E. Treating Helicobacter pylori infection in primary care patients with uninvestigated dyspepsia: the Canadian adult dyspepsia empiric treatment - Helicobacter pylori positive (CADET-Hp) randomised controlled trial. BMJ. 2002;324:1012-1018.

27. Erstad BL. Cost-effectiveness of proton pump inhibitor therapy for acute peptic ulcer-related bleeding. Crit Care Med. 2004;32:1277-1283.

28. Hojo M, Miwa H, Nagahara A, Sato N. Pooled analysis on the efficacy of the second-line treatment regimens for Helicobacter pylori infection. Scand J Gastroenterol. 2001;36:690-700.

29. Howard K, Lord SJ, Speer A, Gibson RN, Padbury R, Kearney B. Value of magnetic resonance cholangiopancreatography in the diagnosis of biliary abnormalities in postcholecystectomy patients: a probabilistic cost-effectiveness analysis of diagnostic strategies. Int J Technol Assess Health Care. 2006;22:109-118.

30. Kuipers EJ. Review article: exploring the link between Helicobacter pylori and gastric cancer. Aliment Pharmacol Ther. 1999;13(Suppl 1): $3-11$.

31. Laine L, Estrada R, Trujillo M, Knigge K, Fennerty MB. Effect of proton pump inhibitor therapy on diagnostic testing for Helicobacter pylori. Ann Intern Med. 1998;129:547-550.

32. Micromedex 2.0 [homepage on the Internet]. Red Book Online. Thomson Reuters; 2014. Available from: http://www.micromedexsolutions.com/ home/dispatch. Accessed March 5, 2015.

33. Slawsky K, Cyr PL, Olchanski N, Howden CW. Cost-effectiveness of diagnostic strategies for $H$. pylori infection in a high prevalence population. Gastroenterology. 2011;140(Suppl 1):S203.

34. Yabroff KR, Lamont EB, Mariotto A, et al. Cost of care for elderly cancer patients in the United States. J Natl Cancer Inst. 2008;100:630-641.

35. Yeh JM, Hur C, Kuntz KM, Ezzati M, Goldie SJ. Cost-effectiveness of treatment and endoscopic surveillance of precancerous lesions to prevent gastric cancer. Cancer. 2010;116:2941-2953.

36. You JH, Tsui KK, Wong RS, Cheng G. Cost-effectiveness of dabigatran versus genotype-guided management of warfarin therapy for stroke prevention in patients with atrial fibrillation. PLoS One. 2012;7:e39640.

37. Whitehead SJ, Ali S. Health outcomes in economic evaluation: the QALY and utilities. Br Med Bull. 2010;96:5-21.

38. Cardenas VM, Mulla ZD, Ortiz M, Graham DY. Iron deficiency and Helicobacter pylori infection in the United States. Am JEpidemiol. 2006; 163:127-134.

39. Niv Y, Hazazi R. Helicobacter pylori recurrence in developed and developing countries: meta-analysis of $13 \mathrm{C}$-urea breath test follow-up after eradication. Helicobacter. 2008;13:56-61.

40. Graham DY, Fishbach L. Recent advances in clinical practice: Helicobacter pylori treatment in the era of increasing antibiotic resistance. Gut. 2010;59:1143-1153. 


\section{Supplementary material}

Table SI Estimation of excess lifetime costs associated with peptic ulcer disease

\begin{tabular}{|c|c|c|}
\hline Calculation step & Equation/notation & Estimate \\
\hline Excess LTCs associated with PUD due to continuing $H$. pylori infection, US\$ & $\mathrm{LTC}_{\text {PUD }}=\left(P_{\text {PUD[W] }}-P_{\text {PUD[WO] }]}\right) \cdot C_{\text {PUD }}$ & 28.09 \\
\hline Expected cost per patient with PUD, US\$ & $C_{\text {PUD }}=C_{\text {med }}+C_{\text {hosp }}$ & 249.65 \\
\hline Expected cost per patient of PUD-related hospitalization, US\$ & $C_{\text {hosp }}=P_{\text {PUD [hosp }]} \cdot C_{\text {PUD [hosp }]}$ & 8.90 \\
\hline Cost of GI hospitalization event due to PUD, US\$ & $C_{\text {PUD[hosp] }}$ & $8,896.00$ \\
\hline Probability of GI hospitalization event due to PUD, \% & $P_{\text {PUD [hosp }]}$ & 0.10 \\
\hline Expected cost per patient of PUD regardless of hospitalization (managed by medication), US\$ & $C_{\text {med }}=t_{\text {PUD [med }]} \cdot C_{\text {PUD }[\text { med }]}$ & 240.75 \\
\hline Duration of PUD, years & $t_{\text {PUD[med] }}$ & 0.50 \\
\hline Cost/year of managing PUD with medication, US\$ & $C_{\text {PUD }[\mathrm{med}]}$ & 481.50 \\
\hline Excess lifetime risk of PUD due to continuing $H$. pylori infection, \% & $P_{P U D[W]}-P_{\text {PUD }[W O]}$ & 11.25 \\
\hline Lifetime risk of developing PUD with continuing $H$. pylori infection, \% & $P_{\text {PUD[W] }}$ & 15.00 \\
\hline Lifetime risk of developing PUD without continuing $H$. pylori infection, \% & $P_{\text {PUD[wo] }}$ & 3.75 \\
\hline
\end{tabular}

Notes: $[w]$ is the condition with $H$. pylori infection; [wo] indicates condition without $H$. pylori infection.

Abbreviations: GI, gastrointestinal; ELTCs, excess lifetime costs; PUD, peptic ulcer disease; Hosp, hospitalization; Med, medication; H. pylori, Helicobacter pyloris.

\section{Publish your work in this journal}

Patient Preference and Adherence is an international, peer-reviewed, open access journal that focuses on the growing importance of patient preference and adherence throughout the therapeutic continuum. Patient satisfaction, acceptability, quality of life, compliance, persistence and their role in developing new therapeutic modalities and compounds to optimize clinical outcomes for existing disease states are major areas of interest for the journal. This journal has been accepted for indexing on PubMed Central. The manuscript management system is completely online and includes a very quick and fair peer-review system, which is all easy to use. Visit http://www. dovepress.com/testimonials.php to read real quotes from published authors.

\footnotetext{
Submit your manuscript here: http://www.dovepress.com/patient-preference-and-adherence-journal
} 